\title{
A variable ultra-luminous X-ray source in the colliding galaxy NGC 7714
}

\author{
R. Soria ${ }^{1,2}$ and C. Motch ${ }^{2}$ \\ 1 Mullard Space Science Laboratory, University College London, Holmbury St Mary, Surrey RH5 6NT, UK \\ e-mail: Roberto.Soria@mssl.ucl.ac.uk \\ 2 Observatoire Astronomique, UA 1280 CNRS, 11 rue de l'Université, 67000 Strasbourg, France \\ e-mail: motch@astro.u-strasbg.fr
}

Received 13 February 2004 / Accepted 23 April 2004

\begin{abstract}
We studied the colliding galaxy NGC 7714 with two XMM-Newton observations, six months apart. The galaxy contains two bright X-ray sources: we show that they have different physical nature. The off-nuclear source is an accreting compact object, one of the brightest ultraluminous X-ray sources (ULXs) found to date. It showed spectral and luminosity changes between the two observations, from a low/soft to a high/hard state; in the high state, it reached $L_{\mathrm{x}} \approx 6 \times 10^{40} \mathrm{erg} \mathrm{s}^{-1}$. Its lightcurve in the high state suggests variability on $\mathrm{a} \approx 2 \mathrm{~h}$ timescale. Its peculiar location, where the tidal bridge between NGC 7714 and NGC 7715 joins the outer stellar ring of NGC 7714, makes it an interesting example of the connection between gas flows in colliding galaxies and ULX formation. The nuclear source $\left(L_{\mathrm{x}} \approx 10^{41} \mathrm{erg} \mathrm{s}^{-1}\right)$ coincides with a starburst region, and is the combination of thin thermal plasma emission and a point-source contribution (with a power-law spectrum). Variability in the power-law component between the two observations hints at the presence of a single, bright point source $\left(L_{\mathrm{x}} \gtrsim 3 \times\right.$ $10^{40} \mathrm{erg} \mathrm{s}^{-1}$ ): either a hidden AGN or another ULX.
\end{abstract}

Key words. black hole physics - galaxies: individual: NGC 7714 - X-rays: galaxies - X-ray: stars - accretion, accretion disks

\section{Introduction}

XMM-Newton and Chandra studies of colliding or merging gas-rich galaxies at various stages of the evolutionary sequence (Toomre 1977) have revealed significant contribution to the X-ray emission both from diffuse hot gas, associated to starburst processes, and from accreting point sources, generally associated to a young stellar population. See, for example: the Mice (Read 2003); the Antennae (Zezas et al. 2002; Fabbiano et al. 2003a); M 82 (Griffiths et al. 2000); the Cartwheel (Gao et al. 2003). A peculiar feature in many of these systems is the presence of accreting X-ray sources brighter than the Eddington limit for a stellar-mass black hole $(\mathrm{BH})\left(L_{\mathrm{Edd}} \approx\right.$ $\left.10^{39} \mathrm{erg} \mathrm{s}^{-1}\right)$; they are commonly known as ultraluminous X-ray sources (ULXs). The ages and masses of the compact objects in ULXs, the nature of the donor stars, and the geometry of emission are still unclear, and hotly debated. It is also unclear precisely why ULXs are preferentially found in interacting galaxies (Swartz et al. 2003), and what this can reveal about their mechanism of formation.

The interacting system Arp 284 (Arp 1966) is an exceptional example of a recent ( 100-200 Myr ago), direct impact (Struck \& Smith 2003). It consists of the nuclear starburst galaxy NGC 7714 (classified as SB(s)b pec ${ }^{1}$ ) and its fainter,

1 NED: NASA Extragalactic Database. currently inactive companion NGC 7715 (Im pec). NGC 7714 is located at a redshift distance of $40.0 \mathrm{Mpc}$ (Huchra et al. 1999, for $\left.H_{0}=70 \mathrm{~km} \mathrm{~s}^{-1} \mathrm{Mpc}^{-1}\right)$. It has a prominent stellar ring, three tidal arms/tails, and is connected to NGC 7715 by a gas and stellar bridge (Arp 1966). Its low inclination (viewing angle of $\left.45^{\circ}\right)$ and low foreground absorption $\left(n_{\mathrm{H}}=4.9 \times\right.$ $10^{20} \mathrm{~cm}^{-2}$; Dickey \& Lockman 1990) make it a good target for $\mathrm{X}$-ray studies.

ROSAT/HRI observations of NGC7714 (Papaderos \& Fricke 1998) have revealed two strong $\left(L_{\mathrm{x}}>10^{40} \mathrm{erg} \mathrm{s}^{-1}\right)$ $\mathrm{X}$-ray sources, separated by $\approx 22^{\prime \prime}$. The brighter one coincides with the starburst nucleus; the fainter one is located approximately where the gas/stellar bridge joins a collisional stellar ring, but does not correlate with any bright counterpart at other wavelengths, nor is it located in a starburst region. It was suggested (Papaderos \& Fricke 1998) that the off-nuclear source might be a compact region of hot shocked gas. This could be due either to the collision of a fast starburst-driven outflow with the colder, denser gas in the galactic bridge; or, to the infall of high-velocity $\mathrm{H}$ I clouds along the bridge onto the outer H I disk of NGC 7714 (a somewhat similar situation to accretion-disk hot spots in X-ray binaries). However, the limited wavelength coverage and resolution of ROSAT did not allow detailed individual analyses of the two sources. 
Table 1. XMM-Newton/EPIC observation log for NGC 7714.

\begin{tabular}{lrrrr}
\hline \hline Date & Obs. ID & Start time & Stop time & $\begin{array}{l}\text { GTI } \\
(\mathrm{ks})\end{array}$ \\
\hline 2002 Jun. 07 & 0112521301 & pn: 10:36:39 & $15: 16: 39$ & 15.8 \\
& & MOS: 10:03:23 & $15: 21: 23$ & 16.1 \\
2002 Dec. 08 & 0112522601 & pn: 12:40:16 & $16: 38: 19$ & 14.3 \\
& & MOS: 12:18:15 & $16: 43: 15$ & 15.9 \\
\hline
\end{tabular}

Here we present some preliminary results of our $X M M-N e w t o n$ study of the system. We argue that the offnuclear source is an accreting point source (in fact, one of the most luminous ULXs ever detected), and we discuss the spectral and timing properties of the two sources.

\section{Observations and data analysis}

NGC 7714 was observed with all instruments on-board XMM-Newton on 2002 June 7 and 2002 December 8 (Table 1); we used the medium filter, full-frame mode for the EPIC detectors. We processed the Observation Data Files with standard tasks in version 5.4 of the Science Analysis System (SAS). After inspecting the background fluxes, we rejected the initial $\approx 1$ ks of the first exposure, which was affected by a background flare: we retained a live-time good-time-interval of $15.9 \mathrm{ks}$ (for the MOSs) and $14.3 \mathrm{ks}$ (for the pn). From the second observation, we used a live-time good-time-interval of $15.8 \mathrm{ks}$ (MOSs) and $12.9 \mathrm{ks}(\mathrm{pn})$. We filtered the event files, selecting only the best-calibrated events (pattern $\leq 12$ for the MOSs, pattern $\leq 4$ for $\mathrm{pn}$ ), and rejecting flagged events. We checked and corrected the astrometry of the various images so that the position of the nuclear source coincided with the NED position (Clements 1983): RA $\left(2000=23^{\mathrm{h}} 36^{\mathrm{m}} 14 \mathrm{~s} .1\right.$, Dec $(2000)=+02^{\circ} 09^{\prime} 18^{\prime \prime} .6$.

Firstly, we extracted source and background spectra for the nucleus and the bright off-nuclear source in NGC 7714, for each detector in each of the two exposures. The off-nuclear source is located at RA $(2000)=23^{\mathrm{h}} 36^{\mathrm{m}} 15^{\mathrm{s}} \cdot 6$, Dec $(2000)=$ $+02^{\circ} 09^{\prime} 23^{\prime \prime}$, , i.e., $\approx 22^{\prime \prime}$ east of the nucleus (Figs. 1 and 2 ). We estimate an error $\lesssim \pm 3^{\prime \prime}$, mostly due to the astrometry uncertainties; however, the error in the relative positions of the nuclear and off-nuclear sources is only $\approx 1^{\prime \prime}$. To reduce the contamination from the bright nearby nucleus, we used as source extraction region a circle of radius $11^{\prime \prime}$. We used as background region the union of three circles of $11^{\prime \prime}$ radius, centred at a distance of $22^{\prime \prime}$ from the nucleus, on the opposite side of the source, so that it would contain a similar contribution from the nuclear source. For the nucleus, we used an extraction circle of radius $15^{\prime \prime}$. The background region was chosen as the union of two circles of radius $15^{\prime \prime}$ centred at $22^{\prime \prime}$ from the nearby off-nuclear source. We tested the effect of different choices of background regions, until we were satisfied that the reciprocal contaminations of the two sources were properly subtracted.

We built response functions for each source with the SAS task rmfgen, and auxiliary response functions with arfgen. Given the small extraction radii used for the two overlapping sources, we corrected for the flux that is outside

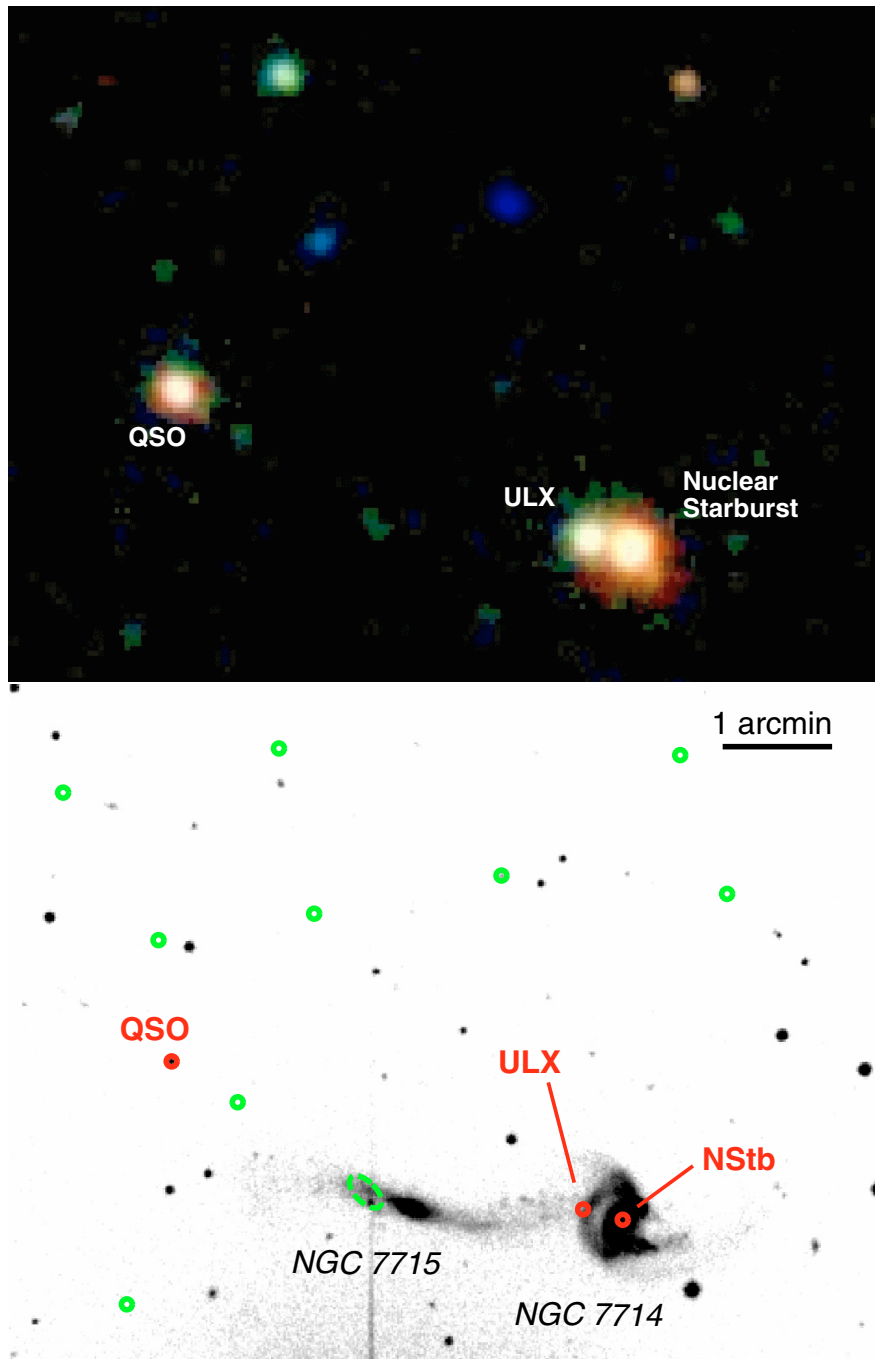

Fig. 1. Top panel: true-color image of the NGC 7714 field obtained by coadding the MOS and pn observations of 2002 Jun. 7 and Dec. 8. The image was smoothed with a $3 \times 3$ pixel boxcar. Red: $0.2-1 \mathrm{keV}$; green: $1-2 \mathrm{keV}$; blue: $2-12 \mathrm{keV}$. Size of the image: $7 ! 8 \times 6 ! 2$; North is up, East is to the left. Bottom panel: on the same scale, the positions of the three brightest X-ray sources in the field are overplotted (red circles) onto a Digitized Sky Survey $B$ image; the positions of a few other, fainter X-ray sources detected with XMM-Newton/EPIC are also overplotted (green circles and ellipse).

the extraction regions by setting "extendedsource = false", "modelee $=$ true" in the arfgen parameters. We then fitted the background-subtracted spectra with standard models in XSPEC v.11.3 (Arnaud 1996); owing to the uncertainties in the EPIC responses at low energies, we used only the $0.3-12 \mathrm{keV}$ range. Firstly, we analysed the individual pn and MOS spectra from each observation. After ascertaining that they were consistent with each other, we coadded them with the method described in Page et al. (2003), in order to increase the signal-tonoise ratio. Thus, we obtained one combined EPIC spectrum of the nucleus and one of the off-nuclear source from each epoch. 
$20 \operatorname{arcsec}$

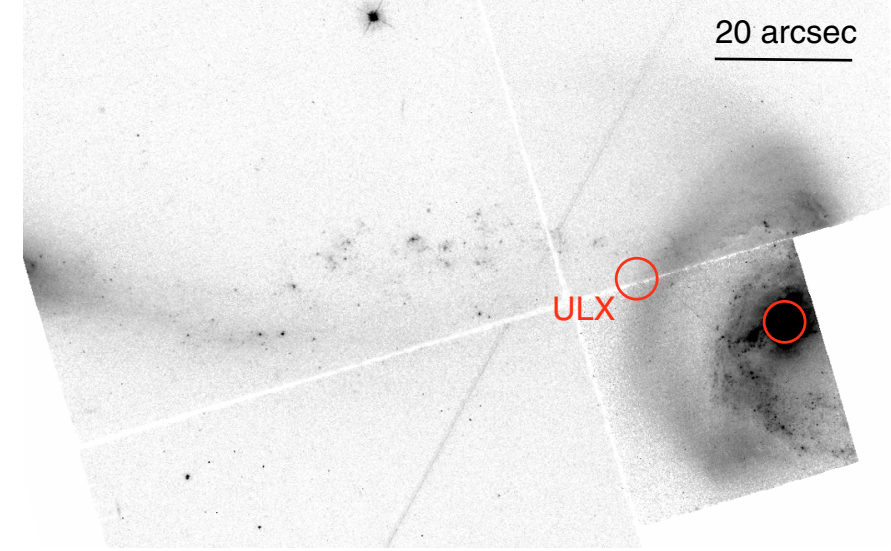

Fig. 2. Position of the ULX and of the X-ray nucleus (red circles with radius $=3^{\prime \prime}$ ) overplotted on an HTS/WFPC 2 image in the $V$ band ( $f 555 w$ filter). The ULX is located where the tidal bridge meets the collisional stellar ring. North is up, East is to the left.

\section{Main results}

\subsection{Starburst nucleus}

The nucleus of NGC 7714 is characterised by an active starburst that started $\sim$ a few $\times 10^{8}$ yr ago, with an average starformation rate $\approx 1 M_{\odot} \mathrm{yr}^{-1}$; the rate has probably been higher by a factor of a few during the most recent burst which started $\approx 5 \mathrm{Myr}$ ago (Lançon et al. 2001). Its optical, UV and IR properties are similar to those of the "prototypical" starburst galaxy M 82. In analogy with other starburst galaxies, we expect the nuclear X-ray emission to be a combination of diffuse hot gas, high-mass X-ray binaries and supernova remnants - including perhaps some contribution from the Type $\mathrm{Ib} / \mathrm{c} \mathrm{SN} 1999 \mathrm{dn}$, located $\approx 13^{\prime \prime}$ south-east of the nucleus (Qiu et al. 1999; Deng et al. 2000).

The limited spatial resolution of XMM-Newton does not allow us to resolve individual sources in the nuclear region. However, we checked whether the observed profile is consistent with the point-spread-function of a point source, using standard tasks in IRAF. We compared the full-width halfmaximum $(F W H M)$ of the radial profile for the nucleus, the offnuclear source and a nearby quasar. Using EPIC/MOS images from the two observations, we obtain that the last two sources have $F W H M \approx 6^{\prime \prime}$, while the nucleus has $F W H M \approx 8^{\prime \prime}$. This suggests that the X-ray emission from the nuclear region is indeed extended, in agreement with the ROSAT/HRI results (Papaderos \& Fricke 1998). No difference in the radial extent of the three sources is found in the EPIC/pn images; however, this is due to the lower spatial resolution and larger pixel size of the pn. We also examined the radial profiles separately for "soft" $(0.2-1.5 \mathrm{keV})$ and "hard" (1.5-12 keV) MOS images. We obtain that, in the hard band, the $F W H M$ is the same for all three objects, consistent with point sources. The larger radial extent of the nuclear source is found only in the soft band, suggesting a different origin for the two components (see Sect. 4.3). Further investigation of the MOS data suggests that the larger radial profile of the nuclear source in the soft band is due to faint emission a few arcsec south-west of the central position, in excess of what would be expected from a point-source pointspread-function. No excess emission is detected east of the central position, suggesting that SN 1999dn does not contribute significantly.

We fitted the nuclear X-ray spectrum with an absorbed two-temperature variable-abundance thermal plasma model (vmekal), plus an absorbed power-law component, which accounts for the contribution from accreting compact objects. We find that we cannot fit the EPIC spectra from 2002 June and December simultaneously with the same set of parameters. The two spectra are identical at energies $\lesssim 1 \mathrm{keV}$, but the flux at higher energies was higher in December. Therefore, we fixed the two thermal-plasma components (which we do not expect to vary on a 6-months' timescale) but allowed the power-law contribution to vary between the two observations. This results in a good fit $\left(\chi_{v}^{2}=0.77\right.$, see Table 2 and Fig. 3). We do not obtain statistically-significant improvements to the fit by allowing different normalisations of the thermal plasma components in the two observations. The total emitted luminosity in the $0.3-12 \mathrm{keV}$ band is $\approx 8 \times 10^{40} \mathrm{erg} \mathrm{s}^{-1}$ in June and $\approx 1.1 \times 10^{41} \mathrm{erg} \mathrm{s}^{-1}$ in December. The fitted temperatures of the optically-thin thermal components are $k T_{1}=0.43_{-0.16}^{+0.10} \mathrm{keV}$ and $k T_{2}=0.98_{-0.15}^{+3.40} \mathrm{keV}$. Thermal plasma emission contributes for $\approx 30-40 \%$ of the emitted luminosity in the $0.3-12 \mathrm{keV}$ band and $\approx 75-80 \%$ in $0.3-1 \mathrm{keV}$ band. The power-law component has a slope $\Gamma \approx 1.9$, consistent both with an AGN spectrum and with typical $\mathrm{X}$-ray binary spectra. The emitted luminosity in the $2-10 \mathrm{keV}$ band is $\approx 2.2 \times 10^{40} \mathrm{erg} \mathrm{s}^{-1}$ and $\approx 3.8 \times 10^{40} \mathrm{erg} \mathrm{s}^{-1}$ in the two epochs. The $2-10 \mathrm{keV}$ luminosity of starburst galaxies can be used to estimate the star-formation rate (Grimm et al. 2003; Ranalli et al. 2003); in particular, a tight relation holds between the star-formation rate and the high-mass $\mathrm{X}$-ray binary (HMXB) contribution to the X-ray luminosity in that band (Gilfanov et al. 2004). A $2-10 \mathrm{keV}$ luminosity $\approx 3 \times 10^{40} \mathrm{erg} \mathrm{s}^{-1}$ corresponds to a star-formation rate of $\approx 3-$ $7 M_{\odot} \mathrm{yr}^{-1}$ (Gilfanov et al. 2004).

As an aside, we also searched for X-ray emission from NGC 7715, the tidally-interacting companion to NGC 7714. Current star-formation from this galaxy is known to be negligible, probably because of a relative lack of gas (Struck \& Smith 2003). We did not significantly detect any source from each observation; however, when we combine both the June and December datasets, we detect a faint, possibly extended source at $\approx 3-\sigma$ level in the EPIC image (Fig. 1). Its location $\left(\right.$ RA $(2000)=23^{\mathrm{h}} 36^{\mathrm{m}} 23^{\mathrm{s}} \cdot 6$, Dec $\left.(2000)=+02^{\circ} 09^{\prime} 32^{\prime \prime}\right)$ puts it in the eastern gas/stellar countertail of NGC 7715 rather than in its nucleus. From the detected count rate, we estimate an average luminosity $\approx 10^{39} \mathrm{erg} \mathrm{s}^{-1}$.

\subsection{Off-nuclear ULX}

We then examined the coadded EPIC spectra of the off-nuclear source from the two observations. It is immediately clear that this source is fundamentally different from the starburst nucleus: its X-ray spectrum is featureless, with no significant evidence of optically-thin plasma emission. It is also clear that the source varied significantly between the two epochs. 
Table 2. Best-fit parameters for the combined EPIC spectra of the nuclear source on 2002 Jun. 7 and Dec. 8. We assumed a Galactic column density $n_{\mathrm{H}, \mathrm{Gal}}=4.9 \times 10^{20} \mathrm{~cm}^{-2}$. We fitted the two spectra simultaneously, assuming that the thermal plasma component did not vary between the two epochs, but leaving the power-law component free to vary. The quoted errors are the $90 \%$ confidence limit. The fluxes are the observed values, the luminosities are the emitted values.

\begin{tabular}{|c|c|c|}
\hline Parameter & Value in Jun. 02 & Value in Dec. 02 \\
\hline \multicolumn{3}{|c|}{ Model: wabs $_{\text {Gal }} \times\left(\right.$ wabs $_{\mathrm{vm}} \times\left(\right.$ vmekal $_{1}+$ vmekal $\left._{2}\right)+$ wabs $_{\mathrm{po}} \times$ po $)$} \\
\hline$n_{\mathrm{H}, \mathrm{vm}}\left(10^{21} \mathrm{~cm}^{-2}\right)$ & \multicolumn{2}{|r|}{$0.8_{-0.3}^{+4.3}$} \\
\hline$n_{\mathrm{H}, \mathrm{po}}\left(10^{21} \mathrm{~cm}^{-2}\right)$ & \multicolumn{2}{|r|}{$1.0_{-0.6}^{+1.0}$} \\
\hline$k T_{\mathrm{vm} 1}(\mathrm{keV})$ & \multicolumn{2}{|r|}{$0.43_{-0.16}^{+0.10}$} \\
\hline$k T_{\mathrm{vm} 2}(\mathrm{keV})$ & \multicolumn{2}{|r|}{$0.98_{-0.15}^{+3.40}$} \\
\hline$K_{\mathrm{vm} 1}\left(10^{-5}\right)$ & \multicolumn{2}{|r|}{$3.6_{-1.5}^{+9.8}$} \\
\hline$K_{\mathrm{vm} 2}\left(10^{-5}\right)$ & \multicolumn{2}{|r|}{$2.0_{-1.4}^{+1.8}$} \\
\hline$\Gamma$ & $2.00_{-0.21}^{+0.22}$ & $1.85_{-0.19}^{+0.19}$ \\
\hline$K_{\mathrm{po}}\left(10^{-5}\right)$ & $4.3_{-1.0}^{+1.4}$ & $6.0_{-1.4}^{+1.6}$ \\
\hline $\mathrm{C}$ & \multicolumn{2}{|r|}{$\left(0.8_{-0.6}^{+0.9}\right)^{a}$} \\
\hline $\mathrm{N}$ & \multicolumn{2}{|r|}{$\left(0.8_{-0.6}^{+0.9}\right)^{a}$} \\
\hline $\mathrm{O}$ & \multicolumn{2}{|r|}{$\left(0.8_{-0.6}^{+0.9}\right)^{a}$} \\
\hline $\mathrm{Ne}$ & \multicolumn{2}{|r|}{$1.2_{-1.2}^{+1.2}$} \\
\hline $\mathrm{Na}$ & \multicolumn{2}{|r|}{$(1.0)$} \\
\hline $\mathrm{Mg}$ & \multicolumn{2}{|r|}{$2.9_{-1.5}^{+2.3}$} \\
\hline $\mathrm{Al}$ & \multicolumn{2}{|r|}{$(1.0)$} \\
\hline $\mathrm{Si}$ & \multicolumn{2}{|r|}{$3.6_{-1.7}^{+5.4}$} \\
\hline$S$ & \multicolumn{2}{|r|}{$(1.0)$} \\
\hline $\mathrm{Ar}$ & \multicolumn{2}{|r|}{$(1.0)$} \\
\hline $\mathrm{Ca}$ & \multicolumn{2}{|r|}{$(1.0)$} \\
\hline $\mathrm{Fe}$ & \multicolumn{2}{|r|}{$(1.0)$} \\
\hline $\mathrm{Ni}$ & \multicolumn{2}{|r|}{$(1.0)$} \\
\hline$\chi_{v}^{2}$ & \multicolumn{2}{|c|}{$0.77(122.4 / 160)$} \\
\hline$f_{0.3-12}\left(10^{-13} \mathrm{erg} \mathrm{cm}^{-2} \mathrm{~s}^{-1}\right)$ & $2.8_{-0.8}^{+0.3}$ & $4.0_{-0.8}^{+0.2}$ \\
\hline$f_{0.3-1}\left(10^{-13} \mathrm{erg} \mathrm{cm}^{-2} \mathrm{~s}^{-1}\right)$ & $0.8_{-0.4}^{+0.1}$ & $0.9_{-0.4}^{+0.1}$ \\
\hline$L_{0.3-12}\left(10^{40} \mathrm{erg} \mathrm{s}^{-1}\right)$ & 7.8 & 10.5 \\
\hline$L_{0.3-12, \mathrm{vm}}\left(10^{40} \mathrm{erg} \mathrm{s}^{-1}\right)$ & \multicolumn{2}{|r|}{2.9} \\
\hline$L_{0.3-12, \mathrm{po}}\left(10^{40} \mathrm{erg} \mathrm{s}^{-1}\right)$ & 4.9 & 7.6 \\
\hline$L_{0.3-1}\left(10^{40} \mathrm{erg} \mathrm{s}^{-1}\right)$ & 3.5 & 3.9 \\
\hline$L_{2-10}\left(10^{40} \mathrm{erg} \mathrm{s}^{-1}\right)$ & 2.2 & 3.8 \\
\hline
\end{tabular}

$a$ We imposed the same abundance for these three elements.

The source cannot be well fitted with a single spectral model in both observations. Limiting our choice to simple models, we find that a multicolor disk-blackbody with $k T_{\text {in }}=$ $1.0 \pm 0.1 \mathrm{keV}$ provides the best fit to the 2002 June spectrum $\left(\chi_{v}^{2}=1.11\right.$; see Table 3 and Fig. 4). A simple power-law fit gives $\Gamma=2.5 \pm 0.2\left(\chi_{v}^{2}=1.20\right)$, but is slightly improved by the addition of a blackbody component at $k T=0.66_{-0.24}^{+0.35} \mathrm{keV}$ $\left(\chi_{v}^{2}=1.15\right)$. On the contrary, a simple power-law model with $\Gamma=2.1_{-0.1}^{+0.2}$ is an excellent fit to the 2002 December spectrum

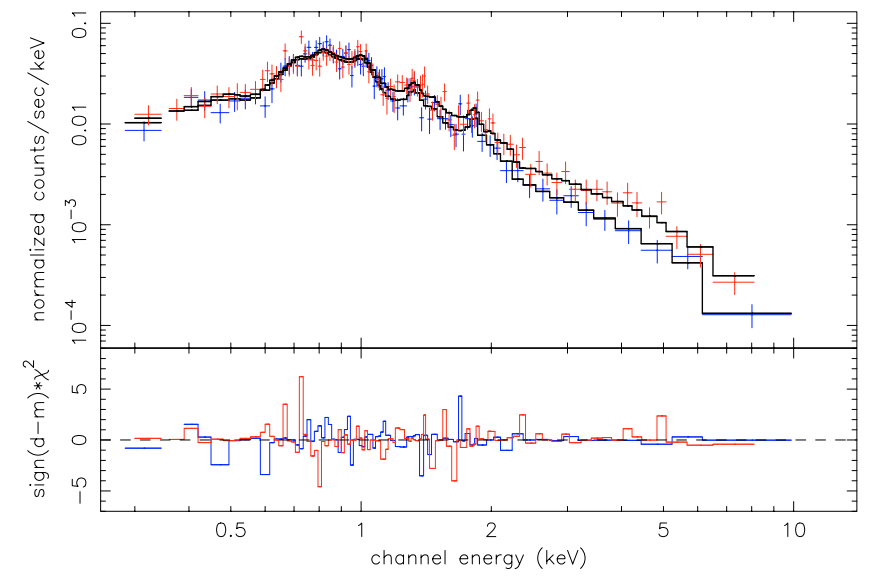

Fig. 3. Coadded EPIC spectra of the nucleus on 2002 Jun. 7 (blue) and Dec. 8 (red), fitted with a constant two-temperature vmekal model plus a varying power-law component.

$\left(\chi_{v}^{2}=0.86\right)$; adding a thermal component does not improve the fit. A multicolor disk-blackbody model is clearly rejected $\left(\chi_{v}^{2}=1.29\right)$ for the second observation. We also tried fitting the two spectra with the Comptonisation model bmc (Shrader \& Titarchuk 1999) (Table 3), confirming that the source was softer in June.

Although any estimates of the emitted luminosity are very model-dependent, it is clear that the source has become brighter by a factor of $\sim 2$ between the two epochs, reaching a luminosity $\approx 6 \times 10^{40} \mathrm{erg} \mathrm{s}^{-1}$ in 2002 December $^{2}$. Such variability on a 6 -month timescale suggests that the size of the emitting region is $\lesssim 0.2 \mathrm{pc}$.

In addition to the long-term variability of the ULX flux, we looked for short-term variations during the two observations. We find that the background-subtracted $0.2-12 \mathrm{keV}$ count rate is consistent with a constant level in the low state. However, variability is detected in the high state (Fig. 5), significant at the $90 \%$ level according to the Kolmogorov-Smirnov test. Fitting the data with a $\sin$ curve gives a period of $6920_{-900}^{+1300} \mathrm{~s}$, and a semi-amplitude of $\approx 18 \%$. However, longer observations are needed to ascertain whether the lightcurve has a real

2 The fact that both the ULX and the nuclear source showed an increase in luminosity from June to December prompted us to doublecheck that this effect is not due to systematic errors. The change in flux is at least an order of magnitude greater than any possible reciprocal contamination between the two sources. Moreover, we verified that the luminosity and spectral variations are consistently seen in the individual pn and MOS spectra; this rules out a systematic error in our coadding of the datasets. In the nucleus, we can clearly attribute the change to the power-law component only, while the thermal plasma emission is unchanged: this also confirms that it is not an instrumental artefact. As a further check, we extracted and analysed the spectra of the nearby QSO [HB89] $2333+019$ (Fig. 1) for the two epochs. We find that the best-fit parameters (power-law with $\Gamma=1.65_{-0.11}^{+0.11}$, and $\Gamma=1.79_{-0.10}^{+0.13}$ respectively) are consistent with an unchanged spectrum. In both observations, the detected QSO flux in the $0.3-12 \mathrm{keV}$ band was $f_{\mathrm{x}}=2.7 \times 10^{-13} \mathrm{erg} \mathrm{cm}^{-2} \mathrm{~s}^{-1}$. We conclude that the variations seen in the ULX and the nucleus between 2002 June and December are real. 
Table 3. Best-fit parameters for the combined EPIC spectra of the ULX on 2002 Jun. 7 and Dec. 8. The quoted errors are the $90 \%$ confidence limit. We assumed a Galactic column density $n_{\mathrm{H}, \mathrm{Gal}}=$ $4.9 \times 10^{20} \mathrm{~cm}^{-2}$. The fluxes are the observed values, the luminosities are the emitted values.

\begin{tabular}{|c|c|c|}
\hline Parameter & Value in Jun. 02 & Value in Dec. 02 \\
\hline \multicolumn{3}{|c|}{ Model: wabs $_{\text {Gal }} \times$ wabs $\times(b b+$ po $)$} \\
\hline$n_{\mathrm{H}}\left(10^{21} \mathrm{~cm}^{-2}\right)$ & $2.2_{-1.1}^{+2.1}$ & $1.5_{-0.4}^{+0.4}$ \\
\hline$k T_{\mathrm{bb}}(\mathrm{keV})$ & $0.66_{-0.24}^{+0.35}$ & - \\
\hline$K_{\mathrm{bb}}\left(10^{-7}\right)$ & $4.4_{-3.5}^{+4.2}$ & - \\
\hline$\Gamma$ & $2.6_{-07}^{+0.4}$ & $2.1_{-0.1}^{+0.2}$ \\
\hline$K_{\text {po }}\left(10^{-5}\right)$ & $3.8_{-1.3}^{+2.3}$ & $6.1_{-0.9}^{+1.1}$ \\
\hline$\chi_{v}^{2}$ & $1.15(43.7 / 38)$ & $0.86(64.8 / 75)$ \\
\hline$f_{0.3-12}\left(10^{-13} \mathrm{erg} \mathrm{cm}^{-2} \mathrm{~s}^{-1}\right)$ & $1.1_{-0.5}^{+0.2}$ & $2.3_{-0.2}^{+0.2}$ \\
\hline$L_{0.3-12}\left(10^{40} \mathrm{erg} \mathrm{s}^{-1}\right)$ & 4.3 & 6.6 \\
\hline \multicolumn{3}{|c|}{ Model: wabs $_{\text {Gal }} \times$ wabs $\times$ diskbb } \\
\hline$n_{\mathrm{H}}\left(10^{21} \mathrm{~cm}^{-2}\right)$ & $0.07_{-0.07}^{+0.49}$ & $<0.12$ \\
\hline$k T_{\text {in }}(\mathrm{keV})$ & $0.97_{-0.13}^{+0.12}$ & $1.07_{-0.10}^{+0.13}$ \\
\hline$K_{\mathrm{dbb}}\left(10^{-3}\right)$ & $6.2_{-2.1}^{+4.9}$ & $7.4_{-2.5}^{+2.6}$ \\
\hline$\chi_{v}^{2}$ & $1.11(44.4 / 40)$ & $1.29(96.7 / 75)$ \\
\hline$f_{0.3-12}\left(10^{-13} \mathrm{erg} \mathrm{cm}^{-2} \mathrm{~s}^{-1}\right)$ & $1.0_{-0.2}^{+0.1}$ & $1.8_{-*}^{+*}$ \\
\hline$L_{0.3-12}\left(10^{40} \mathrm{erg} \mathrm{s}^{-1}\right)$ & 2.3 & 3.8 \\
\hline \multicolumn{3}{|c|}{ Model: wabs $_{\mathrm{Gal}} \times$ wabs $\times$ bmc } \\
\hline$n_{\mathrm{H}}\left(10^{21} \mathrm{~cm}^{-2}\right)$ & $<0.37$ & $0.34_{-0.34}^{+2.9}$ \\
\hline$k T_{\mathrm{bb}}(\mathrm{keV})$ & $0.34_{-0.04}^{+0.04}$ & $0.22_{-0.22}^{+0.11}$ \\
\hline$\Gamma$ & $2.9_{-0.3}^{+0.4}$ & $1.9_{-0.4}^{+0.3}$ \\
\hline $\log (A)$ & $>0.10$ & $>0.21$ \\
\hline$K_{\text {bmc }}\left(10^{-7}\right)$ & $9.4_{-0.8}^{+0.7}$ & $14.5_{-3.4}^{+5.0}$ \\
\hline$\chi_{v}^{2}$ & $1.16(44.1 / 38)$ & $0.87(63.6 / 73)$ \\
\hline$f_{0.3-12}\left(10^{-13} \mathrm{erg} \mathrm{cm}^{-2} \mathrm{~s}^{-1}\right)$ & $1.1_{-*}^{+0.1}$ & $2.4_{-0.6}^{+0.1}$ \\
\hline$L_{0.3-12}\left(10^{40} \mathrm{erg} \mathrm{s}^{-1}\right)$ & 2.4 & 5.1 \\
\hline
\end{tabular}

periodicity. No significant variability is detected from the nucleus within each of the two observations.

\section{Discussion}

\subsection{Intermediate-mass $\mathrm{BH}$ or beamed emission?}

It was suggested (Papaderos \& Fricke 1998) that the off-nuclear $\mathrm{X}$-ray source could be emission from thermal plasma in a hot spot, due either to the collision of a fast outflow with the denser gas in the galactic bridge; or, to the infall of high-velocity clouds along the bridge onto the outer H I disk of NGC 7714. However, both those hypotheses are now ruled out by our $X M M-N e w t o n$ study. From the X-ray spectral properties in the two observations, and from the variability between the two epochs, we conclude that the off-nuclear source is instead an

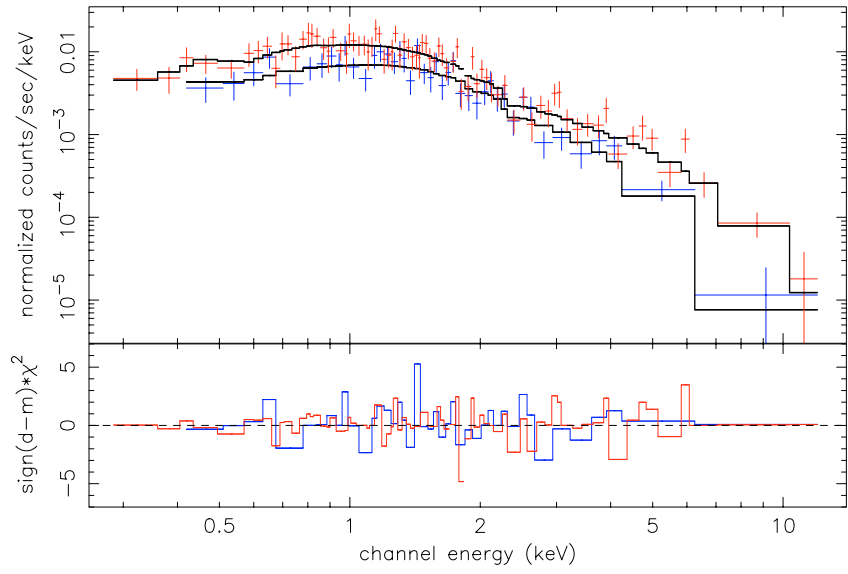

Fig. 4. Coadded EPIC spectra of the ULX on 2002 Jun. 7 and Dec. 8, fitted with a disk-blackbody and a power-law, respectively.

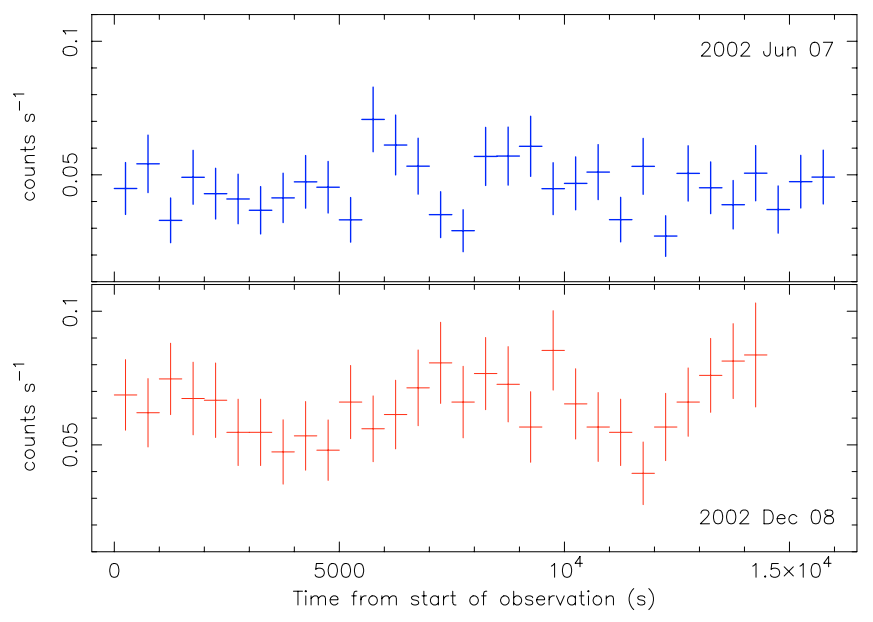

Fig. 5. EPIC lightcurve for the ULX on 2002 Jun. 7 and Dec. 8, binned to $500 \mathrm{~s}$. The background-subtracted count rate is the combined MOS plus pn count rate within an $11^{\prime \prime}$ extraction region; the encircled energy within that radius is $\approx 59 \%$ for MOS 1 , and $\approx 54 \%$ for $\operatorname{MOS} 2$ and pn.

accreting compact object. As such, with an extrapolated bolometric luminosity $\approx 1.5 \times 10^{41} \mathrm{erg} \mathrm{s}^{-1}$ in the high state, it is one of the brightest ULXs ever found. If the emission is isotropic, satisfying the Eddington limit would require a mass of $\approx \mathrm{a}$ few $\times 10^{2} M_{\odot}$ for the accreting $\mathrm{BH}$.

The observed transition between a low/soft and a high/hard spectral state is opposite to what is generally observed in Galactic stellar-mass BH candidates, but similar to what was found for the ULX Holmberg II X-1 (Dewangan et al. 2004) and for some ULXs in the Antennae (Fabbiano et al. 2003b). In the high state, the X-ray spectrum can be modelled by a pure power law with photon index $\Gamma \approx 2$; the conventional explanation for this component is inverse-Compton scattering of soft photons in a hot corona (Sunyaev \& Titarchuk 1980).

A thermal component (blackbody or disk-blackbody) is instead significantly detected in the low/soft state: it contributes for $\approx(0.5-2.3) \times 10^{40} \mathrm{erg} \mathrm{s}^{-1}$ depending on the choice of spectral model; the short duration of our XMM-Newton observations does not allow us to put stronger constraints on the thermal emission. When the low-state spectrum is fitted 
with a disk-blackbody model, the color-temperature parameter $k T_{\text {in }} \approx 1 \mathrm{keV}$ (Table 3 ). If this parameter is identified with the effective temperature at the inner boundary of a ShakuraSunyaev disk (Shakura \& Sunyaev 1973), its high value is inconsistent with the BH mass inferred from the X-ray luminosity. High disk temperatures inconsistent with the standard disk model have been found in many other ULXs (Makishima et al. 2000). They have been explained (Mineshige et al. 2000; Watarai et al. 2001) with a different structure of the accretion flow at high accretion rates ("slim-disk"). Using the slimdisk model, our fitted value of $T_{\text {in }}$ corresponds to a BH mass $\approx 200 M_{\odot}$ (in the Schwarzschild case), with a mass accretion rate $\approx 3 \times 10^{-5} M_{\odot} \mathrm{yr}^{-1}$. Another suggestion put forward to explain the high color temperatures in ULXs is a correction to the disk-blackbody spectrum via a hardening factor $T_{\text {in }} / T_{\text {eff }} \approx 2.6$ (Shrader \& Titarchuk 2003). Finally, a standard accretion disk around a fast-rotating Kerr BH would extend closer to the event horizon and therefore would also have a higher inner temperature than predicted in the non-rotating (Schwarzschild) case (Zhang et al. 1997). However, this effect would be significant only at high inclination angles (edge-on systems) and is unlikely to be the correct explanation for the high-temperature ULX disk spectra (Ebisawa et al. 2003).

An alternative to the intermediate-mass $\mathrm{BH}$ scenario is the possibility that we are looking into a very narrowly collimated beam of emission (beaming factor $\gtrsim 50$ ) from a stellar-mass $\mathrm{X}$-ray binary $\left(\mathrm{BH}\right.$ mass $\lesssim 20 M_{\odot}$ ). Although the latter scenario cannot be ruled out a priori, it would be a very peculiar coincidence to find that the only strongly collimated source aligned - by chance - with our line of sight is located precisely at the junction between the collisional stellar ring and the tidal bridge. A more general, quantitative argument against simple geometrical beaming is discussed in Davis \& Mushotzky (2004), based on the consideration that the scattering region (thick disk or torus) responsible for the beaming can never be a perfect mirror; a large fraction of the radiation would be absorbed and re-radiated isotropically in the optical/IR. Hence, one would expect to find many bright (absolute magnitudes up to $\approx-11$ ) optical/IR point sources for every ULX. Such bright sources have never been seen in nearby galaxies.

Alternatively, the observed ultraluminous flux might be due to relativistic beaming (Urry \& Shafer 1984; Georganopoulos et al. 2002; Körding et al. 2002). In this scenario, the powerlaw component in the X-ray spectrum is the result of inverseCompton scattering of soft photons by the electrons in a jet, moving with velocity $\beta$ and a bulk motion factor $\Gamma=$ $\left(1-\beta^{2}\right)^{-1 / 2}$. Relativistic Doppler boosting implies that the observed luminosity in a given band is enhanced by a factor $[\Gamma(1-\beta \cos \theta)]^{-\alpha}$ where $\alpha \approx 4$. Assuming that the accreting source in NGC 7714 is a stellar-mass $\mathrm{BH}$, and that the mechanical luminosity in the jet is $\lessgtr 0.1 L_{\text {Edd }}$ (e.g., Fender 2001), Lorentz factors $\Gamma \gtrsim 3$ and beaming angles $\lesssim 5^{\circ}$ are required to explain the observed luminosities in the high/hard state. However, this scenario would not explain the soft thermal component seen in the low state, which is unlikely to be beamed.

In conclusion, the location, luminosity and spectral properties of the ULX favour a massive $\left(M>100 M_{\odot}\right)$ accreting object. Further constraints can come from time-variability studies. For example, a break in the power-density spectrum (PDS) at a frequency $\approx\left(M / M_{\odot}\right) \mathrm{Hz}$ has been detected in some AGN and Galactic BH binaries (for recent examples see Markowitz et al. 2003; Uttley et al. 2002; Czerny et al. 2001), and from a ULX in NGC 4559 (Cropper et al. 2004). A preliminary PDS analysis of the ULX in NGC 7714 in its high state suggests features at $\sim 1.5 \times 10^{-4} \mathrm{~Hz}$ (also apparent from the lightcurve in Fig. 5) and $\sim 2 \times 10^{-4} \mathrm{~Hz}$; hence, longer observations could be interesting.

\subsection{ULX formation in colliding galaxies}

One of the few statistically significant properties of the ULX population in nearby galaxies is that these sources are more frequently found in merging or tidally interacting galaxies (Swartz et al. 2003). It is not yet clear why. A simple suggestion is that the ULX formation rate is proportional to the star-formation rate (e.g., Gilfanov et al. 2003), which is known to be higher in interacting galaxies. More specifically, ULX formation could be related to the clustered star-formation rate.

The ULX in NGC 7714 is located on the outer stellar ring, at the junction with the tidal bridge. ULXs are often found in collisional rings and tails of tidally disrupted systems, for example in the outer stellar ring of the Cartwheel galaxy (Gao et al. 2003). However, the two situations are fundamentally different. In the Cartwheel, the outer ring is defined by an expanding wave of star formation (Higdon 1995) associated to a strong density wave in the gas, triggered by the collisional perturbation; hence, ULXs in the Cartwheel ring are clearly associated with active star formation and young stellar clusters. Here, instead, the off-centre collision between the two galaxies produced a weaker dynamical perturbation (Struck \& Smith 2003), and the collisional outer ring is only an expanding density wave of old stars (Bushouse \& Werner 1990), without ongoing star formation: no $\mathrm{H} \alpha$ emission is observed along the ring (González-Delgado et al. 1995; Smith et al. 1997). Radio 21-cm VLA observations also do not show any HI counterpart to the ring (Smith et al. 1997).

Moderately active star formation and HI gas (total HI mass $=1.6 \times 10^{9} M_{\odot}$ ) are instead found along the tidal bridge (Smith et al. 1997). A string of young star clusters along the bridge is also visible in HST/WFPC2 images (Fig. 2; see also Struck \& Smith 2003), although no obvious counterpart is seen in the ULX error circle (this is partly because a WFPC2 chip gap runs exactly across the circle). From the level of $\mathrm{H} \alpha$ emission in the maps of Smith et al. (1997) and González-Delgado et al. (1995), the star-formation rate per unit area in the western part of the bridge (where it joins the stellar ring, near the ULX location) seems to be $\sim 3-3.5$ orders of magnitude lower than in the nuclear starburst region (total $\mathrm{H} \alpha$ luminosity from the bridge $\approx 2 \times 10^{39} \mathrm{erg} \mathrm{cm}^{-2}$ : Smith \& Struck 2001). Assuming that the X-ray luminosity scales with the $\mathrm{H} \alpha$ emission and the star-formation rate (Ranalli et al. 2003; Buat et al. 2002) the extended X-ray emission component from the bridge would be $\lesssim 10^{38} \mathrm{erg} \mathrm{cm}^{-2}$, at least a factor of 10 fainter than our $X M M-N e w t o n / E P I C$ detection limit. Despite the comparatively weaker star-forming activity, the bright ULX is located there 
rather than in the nuclear region. So, the effect of tidal interactions on ULX formation must be more complex than a simple increase of the star-formation rate.

We can clearly rule out the possibility that the ULX be a massive runaway binary formed in the nucleus and then migrated to the outer ring. If, as suggested by its luminosity, the total mass of the binary system is $>25 M_{\odot}$, we expect kick velocities $<10 \mathrm{~km} \mathrm{~s}^{-1}$ (Zezas \& Fabbiano 2002). At a distance of $\approx 4 \mathrm{kpc}$ from the nucleus, it would have taken the ULX $>4 \times 10^{8}$ yr to get there, more than the lifetime of any massive donor stars and possibly more than the age of the nuclear starburst itself (Lançon et al. 2001).

Despite the high column density of atomic hydrogen ( $\gtrsim 10^{21} \mathrm{~cm}^{-2}$ : Smith et al. 1997), CO (1-0) observations failed to detect the tidal bridge (Smith \& Struck 2001). This can be interpreted as evidence for the low metal abundance of the gas. The galactic nucleus is also known to be metalpoor $\left(\sim 0.2-0.4 Z_{\odot}\right.$ : García-Vargas et al. 1997). Other bright ULXs have been found in interacting galaxy systems, located at or near large, high-density $\mathrm{HI}$ or $\mathrm{H}_{2}$ complexes, but with only a weak level of local star formation. For example, the ULX M81 X-9 is associated (Wang 2002) with a molecular cloud or "protogalaxy" near Holmberg IX, with a gas mass $\sim 10^{7} M_{\odot}$ and star-formation rate $\$ 10^{-4} M_{\odot} \mathrm{yr}^{-1}$ (Brouillet et al. 1992; Henkel et al. 1993). In fact, the last close encounter between M 81 and M 82 seems to have left a number of tidal tails and debris (Yun et al. 1994), stripped from the metalpoor outer part of M 81, which are now evolving into tidal dwarf galaxies, with metal abundances $\sim 0.1-0.4 Z_{\odot}$ (Boyce et al. 2001; Makarova et al. 2002).

As pointed out by Pakull \& Mirioni (2002), stellar evolution in metal-poor environments may favor the formation of ULXs, because the mass-loss rate in the radiatively-driven wind from the massive O-star progenitor is much reduced $\left(\dot{M}_{\mathrm{w}} \sim Z^{0.85}\right.$ : Vink et al. 2001; see also Bouret et al. 2003). This leads to a more massive stellar core, which may then collapse into a more massive $\mathrm{BH}$. Hence, it may explain the formation of BHs with masses up to $\approx 50 M_{\odot}$ and isotropic luminosities $\approx 10^{40} \mathrm{erg} \mathrm{s}^{-1}$. Tidal dwarfs, bridges and tails stripped from the outer disks of colliding galaxies may provide an environment for the formation of molecular clouds and stars out of metal-poor gas. However, normal stellar evolution processes alone would be unable to explain accreting BHs with isotropic luminosities $\approx 10^{41} \mathrm{erg} \mathrm{s}^{-1}$ such as the one seen in NGC 7714 .

BHs with masses of $\sim 10^{2}-10^{3} M_{\odot}$ may be formed in the core of young (age $\lesssim 3 \times 10^{6} \mathrm{yr}$ ), massive star clusters, due to the Spitzer instability, runaway core collapse and merger of the O stars (Portegies Zwart \& McMillan 2002; Rasio et al. 2003). Deeper optical observations of the ULX field in this galaxy, and a more precise determination of its position ${ }^{3}$, are needed to investigate the possible association with massive young clusters.

Alternatively, BH remnants in this mass range are thought to be left over by primordial-metallicity (Population III) stars (Madau \& Rees 2001). Most of these BHs could reside in galactic halos: assuming halo masses of $6 \times$ $10^{10} M_{\odot}$ and $2 \times 10^{10} M_{\odot}$ for NGC 7714/15 respectively

\footnotetext{
3 The system has never been observed by Chandra.
}

(Struck \& Smith 2003), and using the results of Islam et al. (2003), one can estimate the presence of $\sim 200$ such intermediate-mass BHs in this system. In an undisturbed galactic halo, accretion from the low-density interstellar medium would make them too faint to be detected. Could galactic collisions and mergers create the conditions for some of these BHs to become bright (up to their Eddington luminosity $\sim 10^{41} \mathrm{erg} \mathrm{s}^{-1}$ ), by providing a fuel supply? This could in principle occur either via tidal capture of a donor star, or via accretion from a dense molecular cloud. The tidal capture timescale can be comparable with the dynamical timescale of the NGC 7714/15 interaction only for stellar densities $\gtrsim 10^{5} \operatorname{stars~pc}^{-3}$ (Fabian et al. 1975; Zezas \& Fabbiano 2002), much larger than in the collisional stellar ring. Hence, this process should be ruled out.

On the other hand, let us consider Bondi-Hoyle accretion from an intergalactic molecular cloud. Assuming an efficiency $\eta=0.1$, an isotropic luminosity $\approx 10^{41} \mathrm{erg} \mathrm{s}^{-1}$ requires an accretion rate $\dot{M} \approx 10^{21} \mathrm{~g} \mathrm{~s}^{-1} \approx 2 \times 10^{-5} M_{\odot} \mathrm{yr}^{-1}$. The Bondi-Hoyle accretion rate (Bondi \& Hoyle 1944; Mirabel et al. 1991) is

$$
\dot{M} \approx 2.4 \times 10^{11}\left(M / M_{\odot}\right)^{2}\left(n / \mathrm{cm}^{3}\right)\left(v / 10 \mathrm{~km} \mathrm{~s}^{-1}\right)^{-3} \mathrm{~g} \mathrm{~s}^{-1},
$$

where $M$ is the mass of the accreting $\mathrm{BH}, n$ is the number density of the molecular gas at large distances from the $\mathrm{BH}$, and $v$ is the velocity of the compact object relative to the cloud. Choosing $M=10^{3} M_{\odot}$ and $v=10 \mathrm{~km} \mathrm{~s}^{-1}, \mathrm{H}_{2}$ number densities $\approx 2 \times 10^{4} \mathrm{~cm}^{-3}$ are required to explain the observed luminosity. Such densities are typical of molecular cloud cores (e.g., Plume et al. 1997), with sizes $\sim 0.1 \mathrm{pc}$. The total molecular gas mass in the NGC 7714 bridge is likely to be $\sim 10^{8}-10^{9} M_{\odot}$, depending on the metal abundance (Smith et al. 1987; Smith \& Struck 2001). Hence, the filling factor of dense molecular cloud cores near the western end of the tidal bridge is only $\sim 10^{-4}$. The probability that a primordial $\mathrm{BH}$ could encounter one such cloud appears to be low. Moreover, the velocity of a halo object relative to the tidal-bridge gas is probably $\gg 10 \mathrm{~km} \mathrm{~s}^{-1}$, making this possibility even less likely. On the other hand, it may be possible that gas has accumulated in the potential well of a massive primordial BH over a long time, before disk instabilities develope and trigger infall into the compact object. Addressing these issues in more details is beyond the scope of this work.

\subsection{Nuclear activity}

Our XMM-Newton study shows that the X-ray spectrum of the starburst nucleus contains a variable power-law component in addition to non-variable thermal plasma emission (Table 2). This suggests that there is probably a single accreting pointsource in the nuclear region contributing a significant fraction of the power-law emission, at least $3 \times 10^{40} \mathrm{erg} \mathrm{s}^{-1}$ in 2002 Dec. It had been speculated in the past that NGC 7714 might contain a low-luminosity AGN, but it was then shown (O'Halloran et al. 2000) that a normal starburst can account for the IR/optical/UV properties. However, these new observations suggest the presence either of an AGN, or of another ULX. 
If the hard source is an AGN, it will slightly "contaminate" our estimate of the star-formation rate (Sect. 3.1). We can gauge this effect by using an alternative relation (Ranalli et al. 2003) between star-formation rate and X-ray luminosity in the $0.5-2 \mathrm{keV}$ band (where the contamination is less significant). Neglecting the power-law component, and considering only the thermal-plasma emission, we infer an emitted luminosity $\approx 2.5 \times 10^{40} \mathrm{erg} \mathrm{s}^{-1}$ in that band. This implies a star-formation rate $\approx 5.5 M_{\odot} \mathrm{yr}^{-1}$; it is a lower limit, since we have ignored the power-law HMXB contribution in that band. Such rate would in turn imply a $2-10 \mathrm{keV}$ luminosity $\approx 2-4 \times 10^{40} \mathrm{erg} \mathrm{s}^{-1}$ (Gilfanov et al. 2004). Therefore, we conclude that the starburst contribution to the $2-10 \mathrm{keV}$ luminosity is $\gtrsim 2 \times 10^{40} \mathrm{erg} \mathrm{s}^{-1}$; the additional contribution (AGN or second ULX) is $\approx 2 \times 10^{40} \mathrm{erg} \mathrm{s}^{-1}$ in the 2002 Dec. observation, and probably negligible in the 2002 Jun. observation. In all cases, the star formation rate is $\sim$ a few $M_{\odot} \mathrm{yr}^{-1}$, in agreement with Lançon et al. (2001).

Other cases of galaxies with variable AGN activity undetected from their optical colors or spectra are discussed in Davis \& Mushotzky (2004) and references therein. A more detailed study of the nuclear starburst, combining the new X-ray data with the multiwavelength results of Lançon et al. (2001), and González-Delgado et al. (1995, 1999), is left to further work.

Finally, we note that the NGC 7714/15 system is one of the examples of alignments between a galaxy and two quasars discussed by H. Arp and collaborators (Stocke \& Arp 1978). Within that scenario, it has been suggested (Arp et al. 2004) that ULXs and quasars might be related to the same physical process of ejection from galactic nuclei. We point out for completeness that the ULX in NGC 7714 is also aligned with the nucleus and the two quasars. However, we have no evidence on which to speculate that this represents more than a coincidence.

\section{Conclusions}

We have used XMM-Newton to study the interacting galaxy system NGC 7714/15. We have reported here the main properties of the two brightest sources: the starburst nucleus and an off-nuclear ULX. The X-ray spectrum of the off-nuclear source suggests that it is an accreting $\mathrm{BH}$, and rules out the possibility that it is due to thermal-plasma emission from a hot spot, as previously speculated. Its X-ray flux varies by a factor of 2 over a six months' interval; the source appears softer in the low state, unlike the typical behaviour of Galactic BH candidates but in agreement with the behaviour of many ULXs. Its spectrum in the low/soft state can be fitted by a disk-blackbody model with $k T_{\text {in }} \approx 1 \mathrm{keV}$ : this is inconsistent with a Shakura-Sunyaev disk, but can be explained with a slim-disk model.

In the high state, its emitted isotropic luminosity is $\approx 6 \times$ $10^{40} \mathrm{erg} \mathrm{s}^{-1}$ in the $0.3-12 \mathrm{keV}$ band, implying a bolometric luminosity $\approx 1.5 \times 10^{41} \mathrm{erg} \mathrm{s}^{-1}$ from a reasonable extrapolation of the power-law spectrum (photon index $\Gamma \approx 2$ ). BH masses of $\sim$ a few $10^{2}-10^{3} M_{\odot}$ would be required to satisfy the Eddington limit. Furthermore, variability on timescales of $\approx 2 \mathrm{~h}$ is detected in the high state.
The ULX is located at the junction of the tidal bridge (consisting of gas and young stars) with the collisional outer ring (consisting of an old stellar population, with no gas). We have pointed out that ULXs are often found in tidally interacting systems, associated with metal-poor molecular clouds, tidal dwarfs, or HI structures formed in the galactic collision.

The nucleus has an X-ray luminosity $\approx 10^{41} \mathrm{erg} \mathrm{s}^{-1}$ in the $0.3-12 \mathrm{keV}$ band. Thermal plasma emission contributes for $\approx 3 \times 10^{40} \mathrm{erg} \mathrm{s}^{-1}$, constant over the two observations, and is probably extended (marginally resolved in the EPIC/MOS images). A point-like power-law component contributes for $\approx 5 \times 10^{40} \mathrm{erg} \mathrm{s}^{-1}$ and $\approx 8 \times 10^{40} \mathrm{erg} \mathrm{s}^{-1}$ in the two observations. The power-law component in the X-ray spectra of starburst nuclei is generally due to unresolved HMXBs. The amount of variability in our case implies that one single source contributes for at least $3 \times 10^{40} \mathrm{erg} \mathrm{s}^{-1}$. This suggests that there is either a hidden AGN or another ULX in the nuclear region.

Acknowledgements. We thank Manfred Pakull for the preparation of the observations and for discussions, and the referee (Curtis Struck) for his useful comments.

\section{References}

Arnaud, K. A. 1996, Astronomical Data Analysis Software and Systems V, ed. G. Jacoby G., \& J. Barnes, ASP Conf. Ser., 101, 17

Arp, H. C. 1966, Atlas of Peculiar Galaxies (Pasadena: Caltech)

Arp, H. C., Gutierrez, C. M., \& Lopez-Corredoira, M. 2004, A\&A, 418,877

Bondi, H., \& Hoyle, F. 1944, MNRAS, 104, 273

Bouret, J.-C., Lanz, T., Hillier, D. J., et al. 2003, ApJ, 595, 1182

Boyce, P. J., Minchin, R. F., Kilborn, V. A., et al. 2001, ApJ, 560, L127

Brouillet, N., Henkel, C., \& Baudry, A. 1992, A\&A, 262, L5

Buat, V., Boselli, A., Gavazzi, G., \& Bonfanti, C. 2002, A\&A, 383, 801

Bushouse, H. A., \& Werner, M. W. 1990, ApJ, 359, 72

Clements, E. D. 1983, MNRAS, 204, 811

Cropper, M. S., Soria, R., Mushotzky, R. F., et al. 2004, MNRAS, 349, 39

Czerny, B., Nikolajuk, M., Piasecki, M., \& Kuraszkiewicz, J. 2001, MNRAS, 325, 865

Davis, D. S., \& Mushotzky, R. F. 2004, ApJ, 604, 653

Deng, J. S., Qiu, Y. L., Hu, J. Y., Hatano, K., \& Branch, D. 2000, ApJ, 540,452

Dewangan, G. C., Miyaji, T., Griffiths, R. E., \& Lehmann, I. 2004, ApJ, 608, L57

Dickey, J. M., \& Lockman, F. J. 1990, ARA\&A, 28, 215

Ebisawa, K., Zycki, P., Kubota, A., Mizuno, T., \& Watarai, K. 2003, ApJ, 597, 780

Fabbiano, G., Krauss, M., Zezas, A., Rots, A., \& Neff, S. 2003a, ApJ, 598, 272

Fabbiano, G., Zezas, A., King, A. R., et al. 2003b, ApJ, 584, L5

Fabian, A. C., Pringle, J. E., \& Rees, M. J. 1975, MNRAS, 172, 15P

Fender, R. P. 2001, MNRAS, 322, 31

Gao, Y., Wang, Q. D., Appleton, P. N., \& Lucas, R. A. 2003, ApJ, 596, L171

García-Vargas, M. L., González-Delgado, R. M., Perez, E., et al. 1997, ApJ, 478, 112

Georganopoulos, M., Aharonian, F. A., \& Kirk, J. G. 2002, A\&A, 388, L25 
Gilfanov, M., Grimm, H.-J., \& Sunyaev, R. 2003, Proc. BeppoSAX Symp., The Restless High-Energy Universe (Amsterdam, May 2003), ed. E. P. J. van den Heuvel, J. J. M. in 't Zand, \& R. A. M. J. Wijers, in press [arXiv: astro-ph/0309725]

Gilfanov, M., Grimm, H.-J., \& Sunyaev, R. 2004, MNRAS, 347, L57

González-Delgado, R. M., García-Vargas, M. L., Goldader, J., Leitherer, C., \& Pasquali, A. 1999, ApJ, 513, 707

González-Delgado, R. M., Pérez, E., Díaz, A. I., et al. 1995, ApJ, 439, 604

Griffiths, R. E., Ptak, A., Feigelson, E. D., et al. 2000, Science, 290, 1325

Grimm, H.-J., Gilfanov, M., \& Sunyaev, R. 2003, MNRAS, 339, 793

Henkel, C., Stickel, M., Salzer, J. J., et al. 1993, A\&A, 273, L15

Higdon, J. L. 1995, ApJ, 455, 524

Huchra, J. P., Vogeley, M. S., \& Geller, M. J. 1999, ApJS, 121, 287

Islam, R. R., Taylor, J. E., \& Silk, J. 2003, MNRAS, 340, 647

Körding, E., Falcke, H., \& Markoff, S. 2002, A\&A, 382, L13

Lançon, A., Goldader, J. D., Leitherer, C., \& González-Delgado, R. M. 2001, ApJ, 552, 150

Madau, P., \& Rees, M. J. 2001, ApJ, 551, L27

Makarova, L. N., Grebel, E. K., Karachentsev, I. D., et al. 2002, A\&A, 396, 473

Makishima, K., Kubota, A., Mizuno, T., et al. 2000, ApJ, 535, 632

Markowitz, A., Edelson, R., Vaughan, S., et al. 2003, ApJ, 593, 96

Mineshige, S., Kawaguchi, T., Takeuchi, M., \& Hayashida, K. 2000, PASJ, 52, 499

Mirabel, I. F., Morris, M., Wink, J., Paul, J., \& Cordier, B. 1991, A\&A, 251, L43

O'Halloran, B., Metcalfe, L., Delaney, M., et al. 2000, A\&A, 360, 871

Page, M. J., Davis, S. W., \& Salvi, N. J. 2003, MNRAS, 343, 1241

Pakull, M. W., \& Mirioni, L. 2002, Proc. Symp. New Visions of the X-ray Universe in the XMM-Newton and Chandra Era, 26-30 November 2001, ESTEC, The Netherlands, in press [arXiv: astro-ph/0202488]
Papaderos, P., \& Fricke, K. J. 1998, A\&A, 338, 31

Plume, R., Jaffe, D. T., Evans, N. J. II, Martin-Pintado, J., \& Gomez-Gonzalez, J. 1997, ApJ, 476, 730

Portegies Zwart, S. F., \& McMillan, S. L. W. 2002, ApJ, 576, 899

Qiu, Y. L., Qiao, Q. Y., \& Hu, J. Y. 2000, IAUC, 7241

Ranalli, P., Comastri, A., \& Setti, G. 2003, A\&A, 399, 39

Rasio, F. A., Freitag, M., \& Gürkan, M. A. 2003, in Carnegie Observatories Astrophysics Series, 1: Coevolution of Black Holes and Galaxies, ed. L. C. Ho (Cambridge: Cambridge Univ. Press)

Read, A. M. 2003, MNRAS, 342, 715

Shakura, N. I., \& Sunyaev, R. A. 1973, A\&A, 24, 337

Shrader, C. R., \& Titarchuk, L. 1999, ApJ, 521, L21

Shrader, C. R., \& Titarchuk, L. 2003, ApJ, 598, 168

Smith, B. J., \& Struck, C. 2001, AJ, 121, 710

Smith, B. J., Struck, C., \& Pogge, R. W. 1997, ApJ, 483, 754

Stocke, J., \& Arp, H. 1978, ApJ, 219, 367

Struck, C., \& Smith, B. J. 2003, ApJ, 589, 157

Sunyaev, R. A., \& Titarchuk, L. G. 1980, A\&A, 86, 121

Swartz, D. A., Ghosh, K. K., \& Tennant, A. F. 2003, Am. Astron. Soc. Meet. 201, 54.13 [arXiv: astro-ph/0302203]

Toomre, A. 1977, ARA\&A, 15, 437

Urry, C. M., \& Shafer, R. A. 1984, ApJ, 280, 569

Uttley, P., McHardy, I. M., \& Papadakis, I. E. 2002, MNRAS, 332, 231

Vink, J. S., de Koter, A., \& Lamers, H. J. G. L. M. 2001, A\&A, 369, 574

Wang, Q. D. 2002, MNRAS, 332, 764

Watarai, K., Mizuno, T., \& Mineshige, S. 2001, ApJ, 549, L77

Yun, M. S., Ho, P. T. P., \& Lo, K. Y. 1994, Nature, 372, 530

Zezas, A., Fabbiano, G., Rots, A. H., \& Murray, S. S. 2002, ApJ, 577, 710

Zhang, S. N., Ebisawa, K., Sunyaev, R., et al. 1997, ApJ, 479, 381 